\title{
Validation of a Low-Cost Electrocardiography (ECG) System for Psychophysiological Research
}

\author{
Ruth Erna Wagner ${ }^{1}\left(\mathbb{D}\right.$, Hugo Plácido da Silva $^{2}\left(\mathbb{D}\right.$ and Klaus Gramann ${ }^{1, *(D)}$ \\ 1 Chair Biological Psychology and Neuroergonomics, TU Berlin, 10623 Berlin, Germany; ruth@wagner2web.eu \\ 2 IT-Instituto de Telecomunicações, 1049-001 Lisbon, Portugal; hsilva@lx.it.pt \\ * Correspondence: klaus.gramann@tu-berlin.de; Tel.: +49-(0)30-314-79-508
}

check for

updates

Citation: Wagner, R.E.; Plácido da Silva, H.; Gramann, K. Validation of a Low-Cost Electrocardiography (ECG) System for Psychophysiological Research. Sensors 2021, 21, 4485. https://doi.org/10.3390/s21134485

Academic Editors: Guruprasad A. Giridharan, Ali H. Mahmoud, Ahmed Shalaby, Ayman El-baz and Mohammed Ghazal

Received: 6 April 2021

Accepted: 28 June 2021

Published: 30 June 2021

Publisher's Note: MDPI stays neutral with regard to jurisdictional claims in published maps and institutional affiliations.

Copyright: (c) 2021 by the authors. Licensee MDPI, Basel, Switzerland. This article is an open access article distributed under the terms and conditions of the Creative Commons Attribution (CC BY) license (https:// creativecommons.org/licenses/by/ $4.0 /)$.

\begin{abstract}
Background and Objective: The reliability of low-cost mobile systems for recording Electrocardiographic (ECG) data is mostly unknown, posing questions regarding the quality of the recorded data and the validity of the extracted physiological parameters. The present study compared the BITalino toolkit with an established medical-grade ECG system (BrainAmp-ExG). Methods: Participants underwent simultaneous ECG recordings with the two instruments while watching pleasant and unpleasant pictures of the "International Affective Picture System" (IAPS). Common ECG parameters were extracted and compared between the two systems. The Intraclass Correlation Coefficients (ICCs) and the Bland-Altman Limits of Agreement (LoA) method served as criteria for measurement agreement. Results: All but one parameter showed an excellent agreement (>80\%) between both devices in the ICC analysis. No criteria for Bland-Altman LoA and bias were found in the literature regarding ECG parameters. Conclusion: The results of the ICC and Bland-Altman methods demonstrate that the BITalino system can be considered as an equivalent recording device for stationary ECG recordings in psychophysiological experiments.
\end{abstract}

Keywords: BITalino; BrainAmp; ICC; intraclass correlation coefficient; Bland-Altman method

\section{Introduction}

In psychophysiological research, Electromyography (EMG), Electrocardiography (ECG), Electrodermal Activity (EDA) and Electroencephalography (EEG) are common electrophysiological methods to investigate the relationship between human behavior and its physiological basis [1,2]. Current instruments are usually stationary, and hence the transmission of the collected data is done by wire, restricting the movement of participants. This is especially detrimental when using such systems in conditions that usually would require movement of participants (e.g., during naturalistic behavior or in virtual reality). Nowadays, there are multiple wearable recording devices on the market. These wearables are mobile and most of them can transmit ECG data wirelessly. However, not all wearables provide access to the data while recording, and they are relatively expensive. Often, proprietary software is necessary for data recording and export for subsequent analyses, adding additional costs and restrictions for the measurement device. Recently, the BITalino has been introduced as an inexpensive hardware and software toolkit specifically designed to deal with the requirements of electrophysiological signal acquisition [3]. The BITalino device transmits data wirelessly and provides the opportunity to access the data while recording.

To ensure that the data recorded with new wearables devices is of sufficient quality to be used in a research context or for non-scientific applications, new devices have to be verified before using them in psychophysiological experiments. Regarding the BITalino, only one study by Carreiras et al. [4] exists in which the BITalino was compared with an established ECG system. However, the main focus of that study was to analyze the morphological similarities between individual heartbeat waveforms and the general similarity between the synchronized time series. Further, the authors used dry electrodes and the 
electrodes were applied to the hand palms or fingers and thus do not represent standard ECG electrode placement. In addition, Carreiras et al. [4] did not use time or frequency domain measures to compare the two devices. While time domain parameters such as heart rate (HR) and heart rate variability (HRV) are established features that can be computed effectively from the ECG, the use of frequency domain parameters provides additional insights into the function of the cardiovascular system [5]. Specifically, the power spectrum of HRV allows for conclusions regarding the involvement of the parasympathetic and sympathetic system in cardiovascular responses. The computation of these features, however, critically depends on the data quality and data processing pipelines, as missing or artifactual beats impact the frequency domain significantly [6-8]. The present study thus used both time and frequency domain parameters to compare the two recording systems and to provide a systematic analyses of ECG parameters.

While the test for concordance of ECG features recorded with two different recording devices could be done based on non-specific ECG signals, the present study used an established psychophysiological protocol to evoke specific ECG activity. We used the "International Affective Picture System" (IAPS) [9] to provoke pronounced differences in ECG features in different test blocks to increase variability in the recordings for a later, more conservative, test for similarity. The IAPS allows for presenting different categories of emotional stimuli (positive, negative and neutral) that are matched regarding their arousal and dominance and that have been used in a large number of psychophysiological studies to evoke different affective responses while controlling for the arousal and dominance associated with a specific affective state [10].

Therefore, the aim of this study was to compare a medical-grade electrocardiography (ECG) system with an ECG sensor of the low-cost DiY (Do-it-Yourself) hardware toolkit BITalino. To evoke clear variation in ECG activity, an experimental protocol inducing different affective states and associated cardiovascular changes was implemented. Several established ECG parameters were extracted from both recordings and tested for similarity between the parameters. Since a statistical test for differences of two or more measures only provides information about differences between conditions, or, as in our case, between two measurement devices, the absence of significance in such tests does not provide evidence for similarity in performance. Correlational measures derived from two different system, in contrast, explain the strength of the relation between two measures but do not indicate their agreement [11]. Testing the agreement or similarity between two measures has to be done using specific statistical approaches that test for the concordance between the measures. There are several methods to test for the concordance of two or more measure with the Intraclass Correlation Coefficients (ICCs) and the Bland-Altman Limits of Agreement (LoA) method representing the most established and tested methods for continuous data with two or more groups [12].

The present study demonstrates that it is possible to reliably record research-grade ECG data with a wearable low-cost device. Reliable data acquisition with a system that does not require proprietary software and can access the recorded data in real time while providing wireless data transmission provides new opportunities for future mobile ECG investigations that do not require expensive laboratory equipment and that allow movement of participants.

The remainder of the work is organized as follows. Section 2 describes the material and methods. Section 3 provides an overview of the ICC and LoA statistical methods. Section 4 summarizes the reliability assessment results. Section 5 presents a discussion and main limitation of the work. Finally, Section 6 outlines the main conclusions and future work perspectives.

\section{Methods}

\subsection{Participants}

Twenty-four participants were recruited through advertisement at the Technical University of Berlin. Only healthy participants without any history of heart disease or pharma- 
cological treatment of heart conditions were accepted. After the recordings, one participant reported to take medication that influenced cardiovascular parameters, leading to the exclusion of this participant. Thus, the final sample consisted of 23 participants (12 women, 11 men) with an age range from 22 to $57(\mathrm{M}=28.3$ years, $\mathrm{SD}=8.8$ years). Participation was voluntary and participants received course credit. They were told not to consume any form of caffeine for $2 \mathrm{~h}$ before the experiment and not to drink alcohol on the day of the experiment. They also got a picture with all electrode positions indicated, so that they could choose appropriate clothing. All subjects gave their consent before being enrolled in the study. The study was approved by the Ethical Commission of IT-Instituto de Telecomunicações with the matriculation number TUB-1234567. Participants provided written informed consent, and the study was conducted in accordance with the Declaration of Helsinki.

\subsubsection{Hardware}

The medical-grade ECG module ExG from BrainProducts was used as the standard ECG system, hereinafter referred to as the BrainAmp-ExG. The BrainAmp-ExG amplifier is an extension available for simultaneous measurement of EEG and other psychophysiological signals such as ECG, EMG and EDA, but it can also be used separately. The BrainAmp-ExG amplifier has a bandwidth from 0 to $1000 \mathrm{~Hz}$. The BrainAmp system is separated from the power grid by the use of a power pack. Electrodes from the BrainAmp-ExG are connected via cables to the amplifier, which is connected to a PC.

As a DiY system, the BITalino version "Plugged BT Kit" was used in the experiment. It contains a control block and sensors for ECG, EMG and EDA, as well as a photo resistor (LUX) and an accelerometer (ACC). The BITalino ECG sensor has a bandwidth between 0.5 and $40 \mathrm{~Hz}$. The electrodes of the BITalino are connected to the BITalino ECG module, which is connected by cable to the BITalino control block. The data can be sent via standard Bluetooth to a recording device. The recording device can be an Android tablet or smartphone, as well as a PC.

\subsubsection{Software}

To allow for a direct comparison of both ECG recordings, the data of the two different amplifier systems were synchronized using the Lab Streaming Layer (LSL) (Christian Kothe, https://github.com/sccn/labstreaminglayer). In Figure 1, the general data acquisition approach is presented. LSL catches data streams in the network, which can be recorded time synchronously by the Lab Recorder software (a part of the LSL package). For providing the data streams to the network, applets were used. The ECG signal from the BrainAmp system is usually recorded with the BrainAmp-Recorder software. An applet for redirecting the data stream from the BrainAmp-Recorder to the network already exists and is part of the LSL package. The applet for the BITalino had to be programmed for this study. The BITalino team provided the BITalino Matlab API, which was added as an official Matlab toolbox [13]. Using that API, the BITalino stream was forwarded to LSL based on the applet for Matlab (9.2.0.538062 (R2017a)).

When different streams are available in the local network, the Lab Recorder detects them and records all selected streams. The Lab Recorder saves the data streams to one file in the open source "extensible data format" (.xdf). XDF is "a general purpose container format for multi-channel time series data with extensive associated meta information. XDF is tailored towards biosignal data such as EEG, EMG, EOG, ECG, GSR, MEG ..." [14]. 


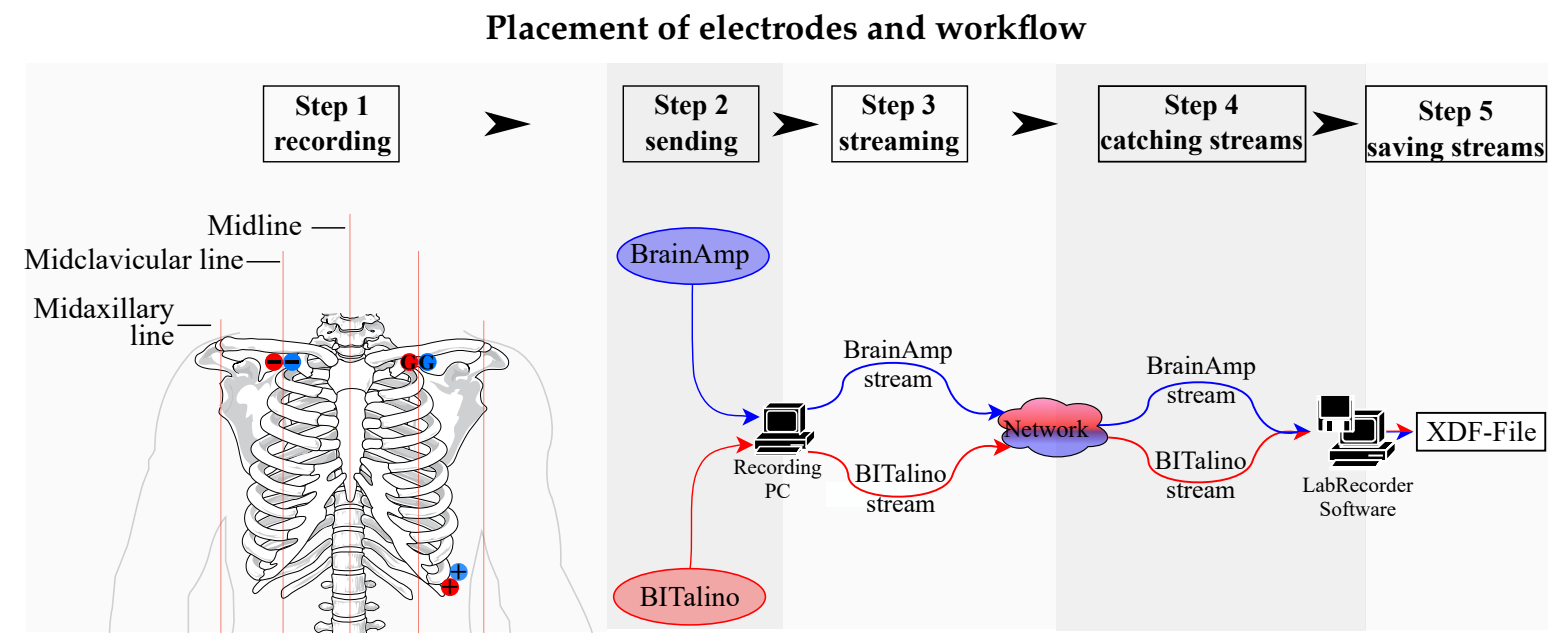

Figure 1. The left side of the image shows the adopted placement of electrodes. The BITalino leads are depicted in red and the BrainAmp leads in blue: - , below right clavicular; +, left side of chest midclavicular line beneath last rib; G, below left clavicular. The right side of the image shows the data acquisition workflow used for recording.

\subsubsection{ECG Electrode Placement and Recording}

A variation of Einthoven lead II was selected for the experiment, referred to as "alternative leads". To allow recordings of the ECG signal concurrently with two devices, electrodes were placed according to the schema presented in Figure 1.

Because ECG is a fast changing signal, a high sampling rate was used. While for the BrainAmp system it is possible to variably set different filters, the filters on the BITalino system are fixed. The BITalino allows data acquisition at sampling rates of 10, 100 and $1000 \mathrm{~Hz}$, and, consequently, a sampling rate of $1000 \mathrm{~Hz}$ was selected for both ECG systems, resulting in one sample per millisecond. In addition, to reduce artifacts in the recordings, high- and low-pass filters were used according to the options available in the two amplifier systems. All settings are listed in Table 1.

Table 1. Hardware filtering specifications and settings used in the experiment. * Note that $0.016 \mathrm{~Hz}=$ time constant of $10 \mathrm{~s}\left(f=\frac{1}{2 \pi c}\right.$, with $f$ the frequency and $c$ a time constant).

\begin{tabular}{lcc}
\hline & BrainAmp & BITalino \\
\hline high-pass filter & $0.016 \mathrm{~Hz}^{*}$ & $0.5 \mathrm{~Hz}$ \\
low-pass filter & $250 \mathrm{~Hz}$ & $40 \mathrm{~Hz}$ \\
sampling rate & $1000 \mathrm{~Hz}$ & $1000 \mathrm{~Hz}$ \\
\hline
\end{tabular}

\subsection{Materials}

Stimuli (IAPS)

The result of this study aimed at establishing whether ECG data recorded with the BITalino system are comparable to an established medical-grade ECG system used in psychophysiological research. To this end, an established psychophysiological paradigm to evoke affective responses that are associated with changes in heart rate was used. Brouwer et al. [15] (p. 3) noted in their study that "most perception studies show valence rather than arousal effects, where pleasant stimuli correlate with higher heart rate acceleration than unpleasant stimuli [16-22]".

We used IAPS pictures to induce two emotional states, pleasant and unpleasant. For the pleasant conditions, pictures with medium arousal ratings were selected, while, for the unpleasant condition, pictures with medium to high arousal ratings were chosen. If the data quality of both the BrainAmp-ExG and the BITalino ECG is comparable, changes in HR dependent on the emotional picture condition should reveal similar values for both systems. 


\subsection{Procedure}

Participants were seated in front of a monitor with a distance of approximately $50 \mathrm{~cm}$ to the screen. They were instructed to sit still for the time of the experimental task, which took $35 \mathrm{~min}$ on average. Stimuli were presented in four blocks (two blocks with pictures of the unpleasant and 2 blocks with pictures of the pleasant condition), consisting of 60 pictures each (Figure 2A). The order of blocks was counterbalanced across participants.

Each picture was presented for $4 \mathrm{~s}$ and stimulus presentations was separated by a fixation cross with $1 \mathrm{~s}$ duration (Figure 2B). After a block of 60 pictures, participants were asked to rate valence and arousal of the entire block on a 10-point scale from 0 to 9 , with 9 indicating the highest arousal or valence, respectively. Before and after two blocks, a 5 min baseline block consisting of a gray fixation cross on black background was added. To control the potential impact of the picture order, pictures were randomly selected from the pool of pictures for each condition and blocks with pleasant and unpleasant pictures were counterbalanced across participants.

After participants were prepared and electrodes were attached, a first baseline block was used for acclimatization. The second baseline block was introduced in the middle of the experiment to recover to the resting heart rate before the last two experimental blocks were presented. After all blocks with picture presentations, a third and final baseline block followed.

A

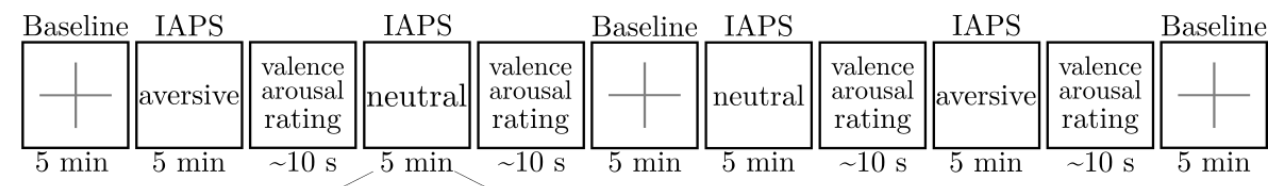

B

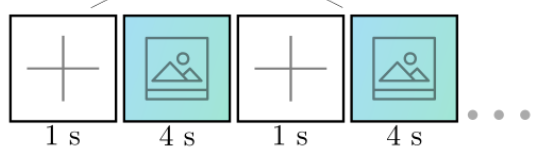

C
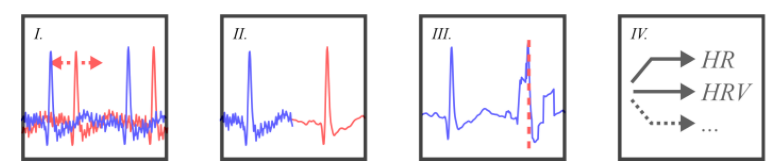

Figure 2. Block diagram of the experimental protocol and analyses pipeline, depicting the stimuli blocks (A), the fixation cross and picture presentation (B), and the overall processing pipeline (C).

\subsection{Data Analysis}

\subsubsection{Data Processing}

Data processing was done in Matlab version 9.2.0.538062 (R2017a) with the use of ECG tool, Matlab-based software developed in-house. The ECG tool offers the option to load .xdf-files. As a result of LSL, both signals were combined with markers in an .xdf-file. Streaming the BITalino data to LSL, after receiving them via Bluetooth from the BITalino devices, had never been tested before. Therefore, visual inspection was done by plotting both signals of one participant in one graph. The overall processing pipeline is depicted in Figure 2C. Due to temporal incongruity of both graphs, alignment was done before data processing. To this end, R-peaks were identified and exported with the corresponding timestamp for each participant. A window of $201 \mathrm{~ms}$ (100 before the R-peak and 100 after the R-peak) was searched to find the timestamp of the corresponding R-peak of the other system. This comparison resulted in differences with a mean difference per participant varying from 19 to $28 \mathrm{~ms}$. The signals were re-synchronized using the mean difference for each participant.

After alignment, the signals from both devices were filtered with a third-order highpass Butterworth filter at $1 \mathrm{~Hz}$ and a third-order low-pass filter at $40 \mathrm{~Hz}$. Then, artifacts were manually identified and subsequently interpolated. For automated R-peak detection 
and to avoid false positives as far as possible, the allowed number of beats per minute (bpm) was set to range between 40 and $125 \mathrm{bpm}$. After automated R-peak detection, false R-peaks were marked and rejected and, in the case of missing peaks, the data were interpolated based on the mean peak interval 5 periods before the missing R-peak.

\subsubsection{Dependent Variables}

According to the Task Force of the European Society of Cardiology and others [23], we computed selected heart rate variability measurements that can be used for shortterm analysis. The aim of the study was to determine whether the BITalino can be used in psychophysiological experiments and, as such, measures were selected which were used in previous experiments. According to Gramann and Schandry [24], the number of heartbeats per minute (designated as heart rate (HR)) is still the most common indicator in psychophysiology to measure cardiovascular events. Heart rate changes accompany almost every change of physical and mental load.

In addition to heart rate measures, measures of heart rate variability were used. For short-term HRV time domain measures, the ref. [23] recommends using RMSSD as an estimate of the short-term components of HRV, which is often used in psychophysiological research. Brouwer et al. [15] also used RMSSD as an estimate for HRV in their study.

In the frequency domain, the ref. [23] recommends three main spectral components for short-term analyses: the very low-frequency (VLF), the low-frequency (LF) and the high-frequency (HF) components. According to the [23] "the distribution of the power and the central frequency of LF and HF are not fixed but may vary in relation to changes in automatic modulations of the heart period ...". The LF component reflects parasympathetic innervation, whereas HF reflects sympathetic and parasympathetic innervation. In addition to this, the ratio between LF and HF (LF/HF) is an indicator of ANS balance. We did not analyze the VLF component as it is not as well defined as the other parameters according to the [23]. In this study, LF, HF and LF/HF ratio were used to investigate whether both systems recorded comparable signals in the experiment.

We expected to see increased HR in blocks with pleasant stimuli as compared to unpleasant stimuli while no directed hypothesis were put forward regarding HRV due to inconsistent results in the literature [16-22,25-28]. While these results would confirm the general validity of our experimental manipulation, the main research question concerned the comparability of the features as measured with the two different ECG systems.

A summary of all measures used in this study can be seen in Table 2.

Table 2. Dependent variables.

\begin{tabular}{llllc}
\hline HR Measures & & HR & Heart Rate & {$[\mathbf{b p m}]$} \\
\hline & time domain & RMSSD & $\begin{array}{l}\text { root mean } \\
\text { square of } \\
\text { successive differences }\end{array}$ & {$[\mathrm{ms}]$} \\
HRV measures & frequency domain & LF & $\begin{array}{l}\text { low frequency } \\
\text { high frequency } \\
\text { ratio between LF and HF }\end{array}$ & {$\left[\mathrm{ms}^{2}\right]$} \\
& & LF/HF & retwen \\
\hline
\end{tabular}

\section{Statistical Methods}

To investigate whether the BITalino system allows for recordings of comparable quality as the established ECG recordings with the BrainAmp system, two methods were used: the Intraclass Correlation Coefficient (ICC) and Bland-Altman Limits of Agreement (LoA) method.

\subsection{Intraclass Correlation Coefficient (ICC)}

According to Müller and Büttner [29] (p. 2465), ICCs are used in medicine to "assess agreement of quantitative measurements in the sense of consistency and conformity". The 
ICC ranges, similar to other correlation coefficients, from 0.00 to 1.00 and is presented in this work as a percentage.

ICCs above $80 \%$ are usually regarded as indicating good to excellent reliability, whereas an ICC between 0.6 and $0.8(60 \%$ and $80 \%)$ may be taken to represent substantial reliability [30]. Portney and Watkins [31] indicated that clinical measurements should show reliability of at least $90 \%$. In addition to the ICC, the lower $95 \%$ confidence interval (lower $\mathrm{CI}$ ) of the ICC can be calculated. Lee et al. [32] reported that an agreement sufficient for the interchangeable use of two methods is suggested only when a lower CI value of $>75 \%$ is observed.

In this study, the ICC form for two-way mixed-effects using single measurements was used to investigate the absolute agreement defined by McGraw and Wong [33]. This approach is mathematically identical to ICC $(2,1)$, as defined by Shrout and Fleiss [34].

\subsection{Bland-Altman Limits of Agreement (Loa) Method}

"The limits of agreement (LoA) method (Altman and Bland [35]; Bland and Altman [11]) for assessing the agreement between two methods of medical measurement is widely used (Bland and Altman [36], Ryan and Woodall [37])" [38] (p. 571).The Bland-Altman method obtains "the differences between measurements by the two methods for each individual" [38] (p. 571) and calculates "the mean and standard deviation" [38] (p. 571). In [38], the authors proposed methods for analyzing repeated data. The LoA were calculated according to the formulas presented in [38].

\section{Results}

\subsection{Descriptive Results}

The HR and HRV parameters in the time and frequency domain revealed only minimal differences in all selected parameters between the two recording devices (Table 3). Moreover, the extracted parameters from both systems during the baseline and the IPAS conditions showed only small differences overall. During blocks with unpleasant stimuli, the heart was lowest for unpleasant followed by pleasant and lastly the baseline blocks. For the RMSSD measures, the lowest HRV was observed during pleasant blocks, followed by unpleasant and baseline blocks. A similar pattern was observed for the ratio LF/HF.

Table 3. Descriptive results.

\begin{tabular}{|c|c|c|c|c|}
\hline & $\begin{array}{l}\text { BITalino } \\
\text { Mean }\end{array}$ & $\begin{array}{c}\text { BrainAmp } \\
\text { Mean }\end{array}$ & $\begin{array}{l}\text { BITalino } \\
\text { SD }\end{array}$ & $\begin{array}{c}\text { BrainAmp } \\
\text { SD }\end{array}$ \\
\hline \multicolumn{5}{|l|}{ HR } \\
\hline Fixation Cross & 73.065 & 73.025 & 10.190 & 10.205 \\
\hline Pleasant & 72.624 & 72.625 & 10.274 & 10.276 \\
\hline Unpleasant & 71.515 & 71.504 & 9.379 & 9.381 \\
\hline \multicolumn{5}{|l|}{ RMSSD } \\
\hline Fixation Cross & 0.045 & 0.045 & 0.025 & 0.025 \\
\hline Pleasant & 0.043 & 0.043 & 0.025 & 0.025 \\
\hline Unpleasant & 0.044 & 0.044 & 0.023 & 0.022 \\
\hline \multicolumn{5}{|l|}{ ratio $\mathrm{LF} / \mathrm{HF}$} \\
\hline Fixation Cross & 3.109 & 2.973 & 3.970 & 3.797 \\
\hline Pleasant & 2.993 & 2.988 & 4.220 & 4.204 \\
\hline Unpleasant & 3.081 & 3.078 & 4.889 & 4.875 \\
\hline
\end{tabular}

\subsection{ANOVA}

A $2 \times 3$ analysis of variance with two levels of the factor "device" (BrainAmp, BITalino) and three levels of the factor "condition" (Baseline (Fixation Cross), pleasant IAPS, unpleasant IAPS) was calculated for all dependent variables. For this analysis, the first fixation block was excluded as it was for acclimatization. Thus, the mean for the factor condition was built of two blocks each. There was a significant main effect of the factor 
"condition" for HR $(\mathrm{F}(1.486,32.685)=4.694, p=0.025)$, as shown in Table 4. For RMSSD, $\mathrm{LF}, \mathrm{HF}$ and LF/HF ratio, the main effect of condition was not significant and there was no interaction effect for "condition $\times$ device" for any dependent variable.

Table 4. Results of $2 \times 3$ ANOVA for the factor "condition" all dependent variables.

\begin{tabular}{clc}
\hline Dependent Variable & Main Factor “Condition” & \\
\hline HR & $F(1.486,32.685)=4.694^{*}$ & $p=0.025^{*}$ \\
RMSSD & $F(2,44)=0.567$ & $p=0.571$ \\
HRV LF & $F(1.200,26.408)=6.204^{*}$ & $p=0.15^{*}$ \\
HRV HF & $F(1,44)=0.127$ & $p=0.881$ \\
HRV LF/HF & $F(1.454,31.997)=0.910^{*}$ & $p=0.384^{*}$ \\
\hline
\end{tabular}

A post hoc pairwise comparison with Bonferroni correction was done for heart rate with an alpha value of 0.05 . None of the pairwise comparisons was significant.

\subsection{Intraclass Correlation Coefficient}

The ICCs and the lower CIs were calculated for all dependent variables over all blocks and, in addition, for each block separately. To gain good to excellent agreement of both devices, the ICC should be higher than $90 \%$ for clinical measurements [31] and the lower CI should be higher than $75 \%$ for the interchangeable use of two methods [32]. The ICC estimates over all blocks for all dependent variables were over $90 \%$ and the lower CIs were over $75 \%$ (Tables 5 and 6). Therefore, all dependent variables met the criterion for good to excellent agreement. For each block separately, the ICC estimates were over $90 \%$ and the lower CIs were over $75 \%$ for all blocks and dependent variables, except for the LF/HF ratio in the second fixation cross (Block 4) (Tables 5 and 6).

Table 5. ICC in percent for each block and dependent variable.

\begin{tabular}{|c|c|c|c|c|c|c|c|c|}
\hline & Overall & $\begin{array}{c}\text { B1 } \\
\text { Fix } 1\end{array}$ & $\begin{array}{l}\text { B2 } \\
\text { P } 1\end{array}$ & $\begin{array}{c}\text { B3 } \\
\text { UP } 1\end{array}$ & $\begin{array}{c}\text { B4 } \\
\text { Fix } 2\end{array}$ & $\begin{array}{l}\text { B5 } \\
\text { P } 2\end{array}$ & $\begin{array}{c}\text { B6 } \\
\text { UP } 2\end{array}$ & $\begin{array}{c}\text { B7 } \\
\text { Fix } 3\end{array}$ \\
\hline HR & $100.0 \%$ & $100.0 \%$ & $100.0 \%$ & $100.0 \%$ & $99.9 \%$ & $100.0 \%$ & $100.0 \%$ & $100.0 \%$ \\
\hline RMSSD & $99.6 \%$ & $99.9 \%$ & $100.0 \%$ & $100.0 \%$ & $97.4 \%$ & $99.8 \%$ & $100.0 \%$ & $100.0 \%$ \\
\hline HRV LF & $100.0 \%$ & $100.0 \%$ & $100.0 \%$ & $100.0 \%$ & $99.9 \%$ & $100.0 \%$ & $100.0 \%$ & $100.0 \%$ \\
\hline HRV HF & $99.6 \%$ & $99.9 \%$ & $99.8 \%$ & $100.0 \%$ & $97.3 \%$ & $99.9 \%$ & $100.0 \%$ & $99.9 \%$ \\
\hline HRV LF/HF & $98.8 \%$ & $100.0 \%$ & $100.0 \%$ & $100.0 \%$ & $83.6 \%$ & $100.0 \%$ & $100.0 \%$ & $99.8 \%$ \\
\hline
\end{tabular}

B1-B7, block number; Fix, fixation cross; P, pleasant IAPS; UP, unpleasant IAPS.

Table 6. Lower CI in percent for each block and dependent variable.

\begin{tabular}{|c|c|c|c|c|c|c|c|c|}
\hline & Overall & $\begin{array}{c}\text { B1 } \\
\text { Fix } 1\end{array}$ & $\begin{array}{l}\text { B2 } \\
\text { P } 1\end{array}$ & $\begin{array}{c}\text { B3 } \\
\text { UP } 1\end{array}$ & $\begin{array}{c}\text { B4 } \\
\text { Fix } 2\end{array}$ & $\begin{array}{l}\text { B5 } \\
\text { P } 2\end{array}$ & $\begin{array}{c}\text { B6 } \\
\text { UP } 2\end{array}$ & $\begin{array}{c}\text { B7 } \\
\text { Fix } 3\end{array}$ \\
\hline HR & $100.0 \%$ & $100.0 \%$ & $100.0 \%$ & $100.0 \%$ & $99.8 \%$ & $100.0 \%$ & $100.0 \%$ & $100.0 \%$ \\
\hline RMSSD & $99.4 \%$ & $99.8 \%$ & $100.0 \%$ & $100.0 \%$ & $94.0 \%$ & $99.6 \%$ & $100.0 \%$ & $99.8 \%$ \\
\hline HRV LF & $99.9 \%$ & $99.9 \%$ & $100.0 \%$ & $100.0 \%$ & $99.8 \%$ & $100.0 \%$ & $100.0 \%$ & $100.0 \%$ \\
\hline HRV HF & $99.4 \%$ & $99.8 \%$ & $100.0 \%$ & $100.0 \%$ & $93.8 \%$ & $99.7 \%$ & $100.0 \%$ & $99.9 \%$ \\
\hline HRV LF/HF & $98.4 \%$ & $99.9 \%$ & $100.0 \%$ & $100.0 \%$ & $65.6 \%$ & $100.0 \%$ & $100.0 \%$ & $99.6 \%$ \\
\hline
\end{tabular}

\subsection{Bland-Altman Method}

An assumption for calculating Bland-Altman bias and limits of agreement is that the difference between both devices are normally distributed. A Shapiro-Wilk test [39] revealed that the data were not normally distributed. Bland and Altman [40] recommended a logarithmic transformation of differences in that case, which also revealed non-normal distribution of the transformed data. Quantile-Quantile plots (Q-Q plots) of the difference between data of the two devices were made to detect the problem on normality tests. The Q-Q plot for the difference of low-frequency measures is shown in Figure 3. 


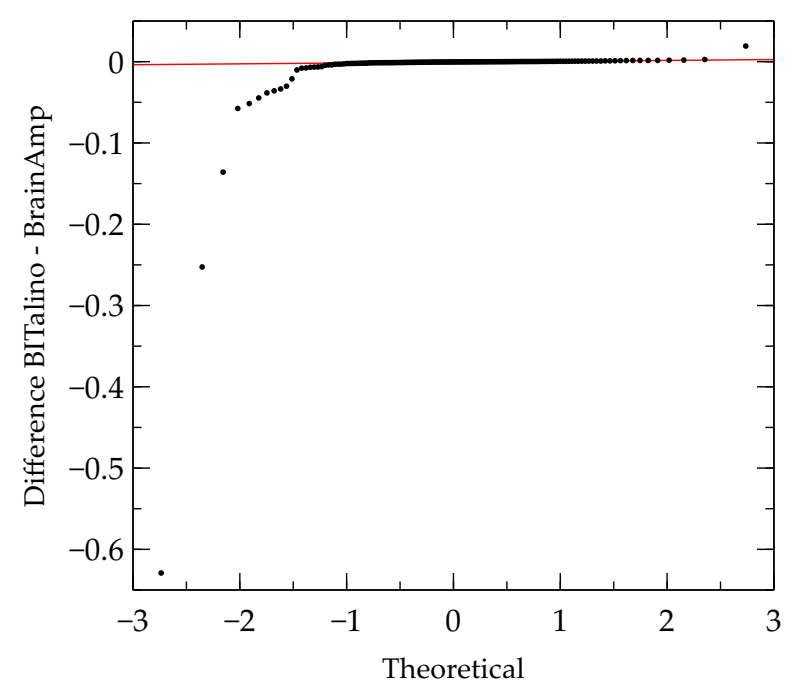

Figure 3. Q-Q plot of differences between the BrainAmp and the BITalino for LF.

In the Q-Q plot, it can be seen that there were several outliers, which may have negatively affected the results of the normality test. Therefore, outliers were excluded and tested again for normality. In some measurements, more than half of the data points had to be excluded to reach normal distribution. However, this heavy reduction of data points may distort the results. According to Bland and Altman [40] (p. 139), a non-normal distribution of the differences values may not be a comparably serious issue for the BlandAltman method as compared to other statistical tests. Non-normal distributed differences will lead to more conservative results than normally distributed differences. For this reason, the non-normal distributed data were used for Bland-Altman analysis even though the normality assumption was violated. The results of the Bland-Altman analysis for non-normal distributed differences are presented in Table 7.

Table 7. Results of Bland-Altman absolute bias and absolute limits of agreement (LoA) for all dependent variables.

\begin{tabular}{lccccc}
\hline Measure & Bias & Lower LoA & - & Upper LoA & Outlier (in \%) \\
\hline HR & -0.01990803 & -0.33662671 & - & 0.29681066 & $4.83 \%$ \\
RMSSD & 0.00003761 & -0.00439510 & - & 0.00447032 & $3.22 \%$ \\
HRV LF & -0.00000880 & -0.00011612 & - & 0.00009852 & $4.83 \%$ \\
HRV HF & 0.00000290 & -0.00019657 & - & 0.00020236 & $4.83 \%$ \\
HRV LF/HF & -0.06054003 & -1.32977327 & - & 1.20869321 & $3.22 \%$ \\
\hline
\end{tabular}

The bias was close to 0 and limits of agreements were quite narrow for all dependent variables. Different results for low and high frequency were found, which may influence the result of the LF/HF ratio negatively. Next, percentages of differences lying outside the LoA were calculated for all dependent variables, as Weippert et al. [41] did in their analysis. The results are presented in Table 7 . The percentage of outliers was lower than $5 \%$ for all measures. Therefore, more than $95 \%$ of differences between the two devices were observed within the limits of agreement. Although the results of Bland-Altmam bias and limits of agreement were quite different, they revealed the same percentage of differences lying outside the limits of agreement.

Visual Inspection of Bland-Altman Plots

Bland and Altman [40] suggested "to plot the difference between the measurements by the two methods for each subject against their mean" (p.140). This kind of plot allows for investigating possible relationships between the discrepancies and the true value. In 
contrast to R-peak analysis, the extracted parameters in the current study were mean values built over a period of time (here over one block). Thus, only seven measures by the two methods were available for each subject. Due to this small amount of measures per subject, Bland-Altman plots of all subjects were created [41]. Percentage of differences lying outside the LoA was under $5 \%$ for each measure, therefore the y-axes of the plots were restricted to the LoA.

The results for Heart Rate (HR) show a bias of $-0.01991 \mathrm{bpm}$ and LoA were at $\pm 0.31672 \mathrm{bpm}$. Therefore, the BITalino yields on average $0.01991 \mathrm{bpm}$ higher values than the BrainAmp-ExG, and the BITalino may yield between $\pm 0.31672 \mathrm{bpm}$ compared to the BrainAmp-ExG. The Bland-Altman plot for HR can be seen in Figure 4.

Due to some extreme data points below 0 , the bias was probably shifted away from 0 to negative. The distribution of data points with the bias of $-0.01991 \mathrm{bpm}$ showed that the BrainAmp was consistently higher than the BITalino for most of the data points.

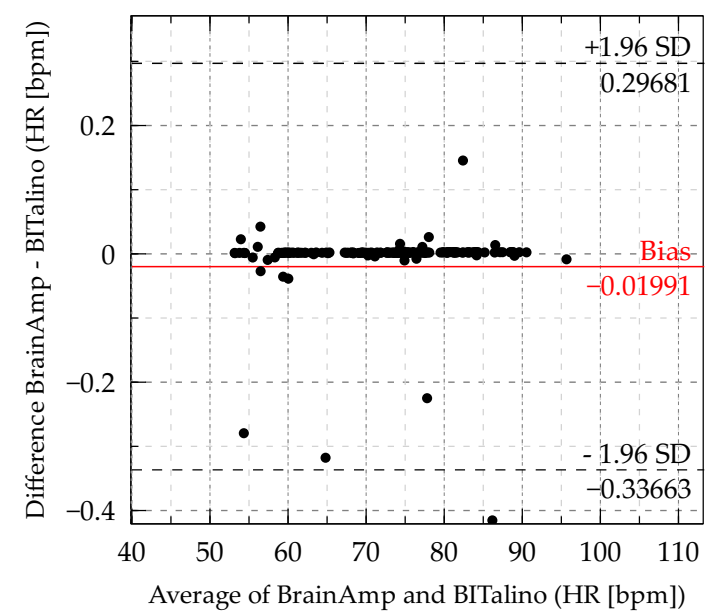

Figure 4. Bland-Altman plot of heart rate. (Some data points outside the LoA are cutoff to offer a more detailed view of the distribution inside the LoA.)

RMSSD. The bias for RMSSD of the complete dataset was at $0.00004 \mathrm{~ms}$ and limits of agreement were at $\pm 0.00443 \mathrm{~ms}$. Thus, the BITalino yielded on average $0.00004 \mathrm{~ms}$ lower values than the the BrainAmp-ExG, and the BITalino may yield $\pm 0.00443 \mathrm{~ms}$ compared to the BrainAmp system. The Bland-Altman plot for RMSSD can be seen in Figure 5.

The distribution of the data showed that most of the data points were near the bias, but that there was a tendency for values of the BrainAmp-ExG to be slightly smaller than the values of the BITalino. Again, limits of agreement were quite narrow despite some extreme values (data points below $-0.00075 \mathrm{~ms}$ and data points above $0.003 \mathrm{~ms}$ ).

Low and high frequency. The bias for LF was at $-0.0088 \mathrm{~ms}^{2}$ and limits of agreement were at $\pm 0.10732 \mathrm{~ms}^{2}$. Thus, the BITalino yields on average $0.0088 \mathrm{~ms}^{2}$ higher values than the BrainAmp, and the BITalino may yield $\pm 0.10732 \mathrm{~ms}^{2}$ compared to the BrainAmp.

The bias for HF was at $0.0029 \mathrm{~ms}^{2}$ and limits of agreement were at $\pm 0.19946 \mathrm{~ms}^{2}$. Thus, the BITalino yields on average $0.0029 \mathrm{~ms}^{2}$ higher values than the BrainAmp, and the BITalino may yield $\pm 0.199 \mathrm{~ms}^{2}$ compared to the BrainAmp. 


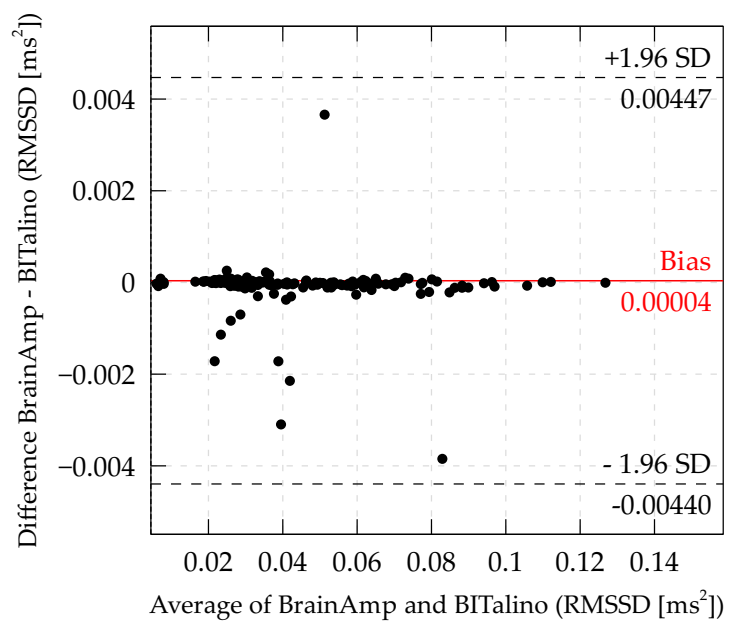

Figure 5. Bland-Altman plot of RMSSD. (Some data points outside the LoA are cutoff to offer a more detailed view of the distribution inside the LoA).

The Bland-Altman plot of high- and low-frequency components of HRV are shown in Figures 6 and 7. In the Bland-Altman plot of the low-frequency component (Figure 6), it can be seen that there were several data points above the bias and a few below the bias. The data points below may have influenced the results of bias and LoA negatively. They may result from interpolated artifacts, which occurred only in one of the systems.

A similar distribution of differences was found for the high-frequency component of HRV (Figure 7). For HF, the bias was positive and most of the data points were below the bias. For both frequency components, there was an increased variability of small values. This may indicate that there is less reliability at small values.

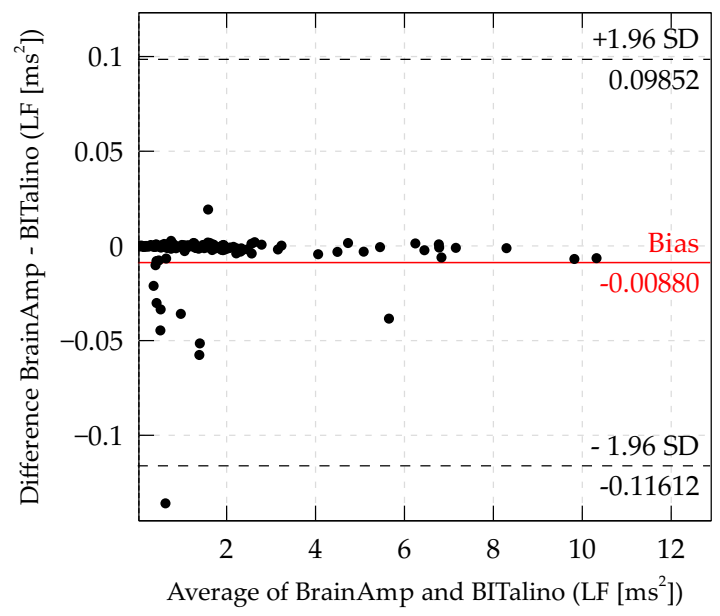

Figure 6. Bland-Altman plot of LF. (Some data points outside the LoA are cutoff to offer a more detailed view of the distribution inside the LoA.) 


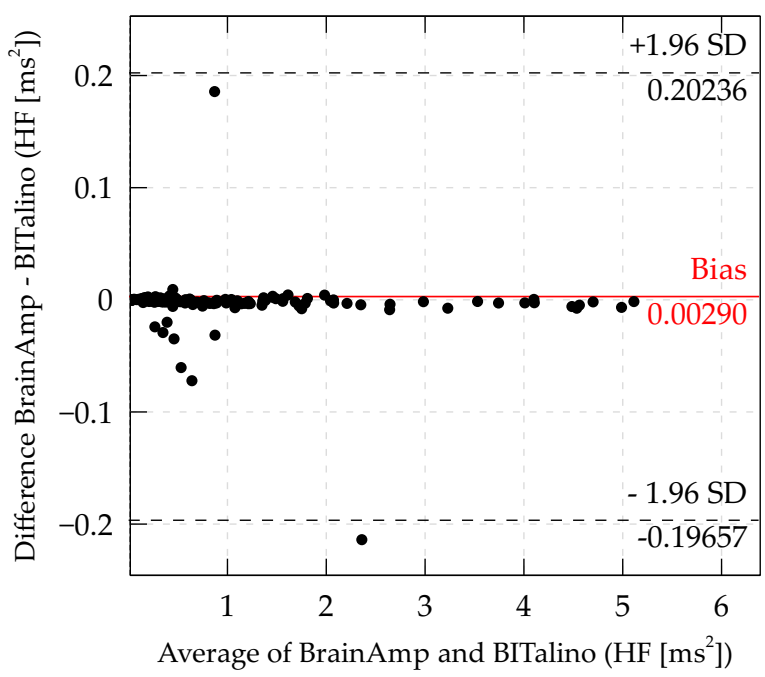

Figure 7. Bland-Altman plot of HF. (Some data points outside the LoA are cutoff to offer a more detailed view of the distribution inside the LoA.)

LF/HF ratio. The bias for LF/HF was at -0.06054 and limits of agreement were \pm 1.26923 , which was the largest bias and widest LoA of all measures. Therefore, the Bland-Altman plot was inspected for LF/HF ratio too (Figure 8).

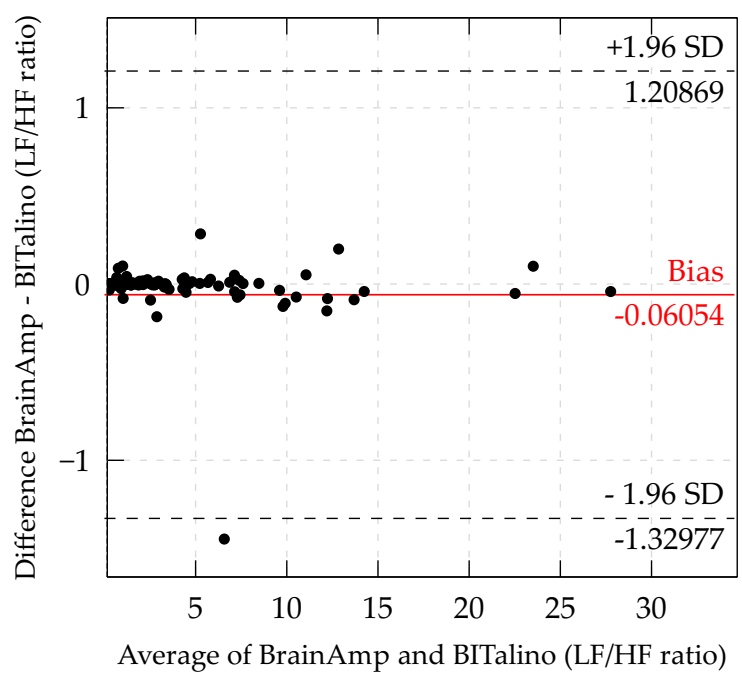

Figure 8. Bland-Altman plot of LF/HF ratio. (Some data points out- side the LoA are cutoff to offer a more detailed view of the distribution inside the LoA.)

Although all the parameters have different measurement scales, differences between measurement devices should always be close to 0 . Therefore, a comparison of limits of agreement between measurements can be done. For the LF/HF ratio, the limits of agreement were 6 times larger than for the HF and 11 times larger than for the LF. Due to the fact that the ratio is built by both components, artifacts may influence the LF/HF ratio even more than both frequency components separately. One extreme difference of -8 (this point is not shown in the graph) may be the result of artifact interpolation in one of the measurements. Both frequency components showed agreement between the measurement devices. However, these different results may indicate that there is probably no or little agreement for the ratio of LF and HF. 


\section{Discussion}

\subsection{Overall ICC and Bland-Altman Method}

All blocks were used in the overall ICC and Bland-Altman analysis. The results reveal that all measures show good to excellent agreement with the ICC method when using the criterion for clinical measurements (ICC > 90\%). This result is consistent with the result of the study by Sandercock et al. [42], who found good to excellent agreement for all measures (LF, LF(nu), HF, HF(nu), LF:HF, RMSSD, SDNN and Mean R- R) when comparing different devices. They also used the Bland-Altman method in addition to the ICC.

In contrast to the ICC, Sandercock et al. [42] found no acceptable agreement between three instruments in the Bland-Altman analysis. They found one acceptable BlandAltman result for the high frequency band of the HRV in one condition for two of the three instruments. They found a bias of $-1 \mathrm{~ms}$ and a LoA of $\pm 264.6 \mathrm{~ms}$. In contrast to Sandercock et al. [42], the present study analyzed high and low frequency measures of $\mathrm{HRV}$ in $\mathrm{ms}^{2}$ and not in $\mathrm{ms}$.

The results of the analyses demonstrate a bias for the high-frequency components of the HRV with $0.0029 \mathrm{~ms}^{2}$ and LoA at $\pm 0.19946 \mathrm{~ms}^{2}$. Similar results were found for the low-frequency component of HRV with a bias of $-0.0088 \mathrm{~ms}^{2}$ and a LoA of $\pm 0.10732 \mathrm{~ms}^{2}$. Based on the results and comparison with previous studies, it can be concluded that the low- and high-frequency components of HRV measured with the BITalino and the BrainAmpp-ExG showed a high level of agreement between the two systems.

In addition, the limits of agreement were more conservative for these non-normally distributed differences than for normally distributed differences.

\subsection{ICC for Each Block}

All measures showed a good to excellent agreement in all blocks except the ratio of $\mathrm{LF} / \mathrm{HF}$ in the second baseline block (Block 4). The second unpleasant IAPS block (Block 3) and the second baseline block (Block 4) each contained more than 40 a of artifacts only in the BrainAmp recordings. However, the LF/HF showed 100\% agreement in Block 3 but only $83.6 \%$ in Block 4 . This might indicate that there was no evidence of artifact interpolation to influence the result of the ICC. The poor agreement of the LF/HF ratio in the second baseline block (Block 4) may be the result of movement, as participants had to sit still already for $15 \mathrm{~min}$ when beginning with the fourth block. Due to the fact that Bland-Altman uses all blocks as repeated measurements, the poor agreement for Block 4 may explain the wider limits of the LF/HF ratio in Bland-Altman analysis.

\subsection{Conclusion of Method Comparison}

As mentioned above, ICC and Bland-Altman analysis as clinical measurements are discussed controversially in the literature. Most of the researched studies investigating ECG comparisons $[42,43]$ used both methods. In the case of Bland-Altman analyses, calculations depended on the design of the study. In the present study, measures of different blocks were used as repeated measurements. None of the referenced comparison studies described which Bland-Altman calculation was used. In addition, criteria for accepting both devices as interchangeable were not mentioned in these studies. For the ICC method, criteria were defined and therefore the interpretation of the results shows higher validity as compared to the Bland-Altman analysis.

Evaluating both methods and comparing their results, as well as inspecting BlandAltman plots, allowed for an objective conclusion about the agreement of measures based on the two systems. Unfortunately, there are no guidelines for a Bland-Altman plot inspection. As demonstrated for the heart rate measure, scaling of the y-axis may mislead the interpretation of the distribution of differences. Furthermore, in the current study, the differences of all measures were not normally distributed, nor were the logarithmic transformed differences normally distributed. Nevertheless, Bland-Altman analysis was computed for the non logarithmic transformed and non-normal distributed data, violating the assumptions of distribution values bias and LoA. 
ICC showed good to excellent agreement, Bland-Altman bias was small and LoA were narrow for almost all variables. The bigger bias and LoA for LF/HF may be explained by the influence of artifacts on one of the devices. If there is a big difference in LF and a small difference in HF, the ratio will be large and vice versa. However, taking all results, the data provide good evidence that both instruments showed very good agreement and can be used in further experiments interchangeably.

\subsection{Limitation of Comparison Methods in the Current Study}

The study was carried out with a sample composed of healthy participants, spanning an age range between 22 and 57 years old. To further encompass the variance that studies in psychophysiology require, future work should be developed focusing on replicating the current study for other sample profiles. Nevertheless, the critical comparison of the two systems was a within-subject design that should not be influenced by a restricted sample profile. The current study is in line with the state-of-the-art, in terms of sample size, and demonstrates the validity of the low-cost system under analysis for the sample enrolled in the study.

Integrating the BITalino stream via LSL required the use of an applet. In the current study, signals of both systems were not aligned. The validity of synchronously acquired data was already demonstrated by da Silva et al. [44]. This non-constant difference between R-peaks of both devices may arise from the working memory load of the Matlab-applet receiving data from the BITalino and forwarding it as a data stream over the network to LSL. The applet for receiving and forwarding the signal from the BITalino to LSL may be implemented in another programming language for future experiments to avoid this non-constant shift.

Furthermore, the BrainAmp ECG was used in comparison as the standard method. However, $93.52 \%$ of artifacts were found in the signals of the BrainAmp ECG. Different leads were used for the BrainAmp and the BITalino, because of the different connection of the leads to the specific system. The BrainAmp leads had been used very often before this experiment which might may have caused some material degradation and, as a consequence, worse signal quality as compared to the new BITalino sensors. Therefore, the BrainAmp-ExG leads had a higher sensitivity to movements and resulting movement artifacts. In the pretests before the experiments, both devices showed no artifacts and therefore the same leads were used in the experiment.

\section{Conclusions and Future Work}

Due to the comparison results of ICC and the Bland-Altman method, the BITalino can be considered as an equivalent recording device for stationary ECG recordings in psychophysiological experiments. The applet to stream the data will be implemented in another programming language and will be tested for future experiments. A new version of the BITalino called "(r)evolution" was introduced in the middle of 2017. Cable plugs were improved (from Molex Sherlock connectors to USB-like UC-E6 connectors); WiFi and BLE (Bluetooth Low Energy or Bluetooth 4.0) were added as technologies for data transmission; and new sensors were introduced. The first BITalino used Molex Sherlock connectors for the electrode leads, which was highly sensitive to cable movement. This may be fixed with the new UC-E6 plugs. The connectivity for data transmission was limited to Bluetooth 2.0. With the new version of BITalino, data can also be sent via BLE or $\mathrm{WiFi}$, which can be advantageous in several use cases. This may fix some of the problems associated with receiving data from the BITalino in the current study. The improvements of the new BITalino (r)evolution and the sensors will be tested in further experiments, following the procedure of the current study. Overall, the results of the present study demonstrate good agreement between an inexpensive DiY system and an established medical grade ECG system. This provides a basis for the BITalino to be used for research in the lab and due to its portability, potentially mobile ECG system outside the lab. 
Author Contributions: Conceptualization, R.E.W. and K.G.; methodology, R.E.W., H.P.d.S. and K.G.; formal analysis, R.E.W.; investigation, R.E.W.; and supervision, H.P.d.S. and K.G. All authors have read and agreed to the published version of the manuscript.

Funding: This work was supported in part by the Ministry of Economy and Competitiveness of the Spanish Government co-founded by the ERDF (PhysComp project) under Grant TIN2017-85409$\mathrm{P}$, and in part by the Instituto de Telecomunicaç oes (IT) in the scope of program under Grant UID/EEA/50008/2019.

Institutional Review Board Statement: The study was conducted according to the guidelines of the Declaration of Helsinki, and approved by the Ethics Committee of IT—Instituto de Telecomunicações (protocol code TUB-1234567, April 2021).

Informed Consent Statement: Informed consent was obtained from all subjects involved in the study.

Data Availability Statement: The data presented in this study are available upon reasonable request made to the corresponding author. The data are not publicly available due to privacy restrictions.

Acknowledgments: The authors would like to thank Ole Traupe from the department of Biological Psychology and Neuroergonomics at the Technical University of Berlin, for the developed Matlabbased software that supports part of the experimental component.

Conflicts of Interest: The funders had no role in the design of the study, in the collection, analyses, or interpretation of data, in the writing of the manuscript, or in the decision to publish the results. H.S. is one of the creators of the BITalino platform and one of the co-founders of the company currently commercializing it.

$\begin{array}{ll}\text { Abbreviations } \\ \text { The following abbreviations are used in this manuscript: } \\ \text { ACC } & \text { accelerometer } \\ \text { ANS } & \text { autonomic nervous system } \\ \text { API } & \text { application programming interface } \\ \text { BLE } & \text { Bluetooth Low Energy/Bluetooth } 4.0 \\ \text { Bpm/bpm } & \text { beats per minute } \\ \text { CI } & \text { confidence interval } \\ \text { DiY } & \text { do-it-yourself } \\ \text { ECG } & \text { electrocardiography } \\ \text { EDA } & \text { electrodermal activity } \\ \text { EEG } & \text { electroencephalography } \\ \text { EMG } & \text { electromyography } \\ \text { EOG } & \text { electrooculography } \\ \text { GSR } & \text { galvanic skin response } \\ \text { HF } & \text { High Frequency } \\ \text { HF(nu) } & \text { High Frequency normalized unit } \\ \text { HR } & \text { Heart Rate } \\ \text { HRV } & \text { heart rate variability } \\ \text { Hz } & \text { Hertz } \\ \text { IAPS } & \text { International Affective Picture System } \\ \text { ICC } & \text { Intraclass Correlation Coefficient } \\ \text { LF } & \text { Low Frequency } \\ \text { LF(nu) } & \text { Low Frequency normalized unit } \\ \text { LF/HF } & \text { ratio between low frequency and high frequency } \\ \text { LoA } & \text { Limits of Agreement } \\ \text { LSL } & \text { Lab Streaming Layer } \\ \text { LUX } & \text { photo transistor } \\ & \end{array}$




$\begin{array}{ll}\text { MEG } & \text { magnetoencephalography } \\ \text { Q-Q plot } & \text { quantile-quantile plot } \\ \text { RMSSD } & \text { Root Mean Square of Successive Differences } \\ \text { SDNN } & \text { Standard deviation of the NN (R-R) intervals } \\ \text { USB } & \text { Universal Serial Bus } \\ \text { VLF } & \text { very low frequency } \\ \text { XDF } & \text { extensible data format }\end{array}$

\section{References}

1. Jimenez-Molina, A.; Retamal, C.; Lira, H. Using psychophysiological sensors to assess mental workload during web browsing. Sensors 2018, 18, 458. [CrossRef] [PubMed]

2. Manzey, D.; Luz, M.; Mueller, S.; Dietz, A.; Meixensberger, J.; Strauss, G. Automation in surgery: The impact of navigated-control assistance on performance, workload, situation awareness, and acquisition of surgical skills. Hum. Factors 2011, 53, 584-599. [CrossRef] [PubMed]

3. Da Silva, H.P.; Fred, A.; Martins, R. Biosignals for Everyone. IEEE Pervasive Comput. 2014, 13, 64-71. [CrossRef]

4. Carreiras, C.; Lourenço, A.; Silva, H.; Fred, A. Comparative Study of Medical-grade and Off-the-Person ECG Systems. In Proceedings of the International Congress on Cardiovascular Technologies-IWoPE (CARDIOTECHNIX), Vilamoura, Portugal, 20-21 September 2013; INSTICC, SciTePress: Setúbal, Portugal, 2013; pp. 115-120. [CrossRef]

5. Ishaque, S.; Khan, N.; Krishnan, S. Trends in Heart-Rate Variability Signal Analysis. Front. Digit. Health 2021, 3, 13. [CrossRef]

6. Baek, H.J.; Shin, J. Effect of missing inter-beat interval data on heart rate variability analysis using wrist-worn wearables. J. Med. Syst. 2017, 41, 1-9. [CrossRef] [PubMed]

7. Morelli, D.; Rossi, A.; Cairo, M.; Clifton, D.A. Analysis of the impact of interpolation methods of missing RR-intervals caused by motion artifacts on HRV features estimations. Sensors 2019, 19, 3163. [CrossRef] [PubMed]

8. Clifford, G.D.; Tarassenko, L. Quantifying errors in spectral estimates of HRV due to beat replacement and resampling. IEEE Trans. Biomed. Eng. 2005, 52, 630-638. [CrossRef]

9. Lang, P.J.; Bradley, M.M.; Cuthbert, B.N. International Affective Picture System (IAPS): Affective Ratings of Pictures and Instruction Manual; Technical Report A-8; Technical Report; The Center for the Study of Emotion and Attention (CSEA): Gainesville, FL, USA, 2008.

10. Bota, P.J.; Wang, C.; Fred, A.L.; Da Silva, H.P. A review, current challenges, and future possibilities on emotion recognition using machine learning and physiological signals. IEEE Access 2019, 7, 140990-141020. [CrossRef]

11. Bland, J.M.; Altman, D. Statistical methods for assessing agreement between two methods of clinical measurement. Lancet 1986, 327, 307-310. [CrossRef]

12. Ranganathan, P.; Pramesh, C.; Aggarwal, R. Common pitfalls in statistical analysis: Measures of agreement. Perspect. Clin. Res. 2017, 8, 187. [CrossRef]

13. Team, M.I.C.T. BITalino Toolbox-File Exchange-MATLAB Central. 2018. Available online: https://ww2.mathworks.cn/ matlabcentral/ fileexchange/53983-bitalino-toolbox?requestedDomain=zh (accessed on 2 June 2021).

14. Kothe, C.; Brunner, C. Extensible Data Format (XDF); Swartz Center for Computational Neuroscience (SCCN), University of California: San Diego, CA, USA, 2018.

15. Brouwer, A.M.; van Wouwe, N.; Mühl, C.; van Erp, J.; Toet, A. Perceiving blocks of emotional pictures and sounds: Effects on physiological variables. Front. Hum. Neurosci. 2013, 7. [CrossRef]

16. Greenwald, M.K.; Cook, E.W.; Lang, P.J. Affective judgment and psychophysiological response: Dimensional covariation in the evaluation of pictorial stimuli. J. Psychophysiol. 1989, 3, 51-64.

17. Bradley, M.M.; Cuthbert, B.N.; Lang, P.J. Startle Reflex Modification: Emotion or Attention? Psychophysiology 1990, $27,513-522$. [CrossRef]

18. Lang, P.J.; Bradley, M.M.; Cuthbert, B.N. Emotion, motivation, and anxiety: Brain mechanisms and psychophysiology. Biol. Psychiatry 1998, 44, 1248-1263. [CrossRef]

19. Bradley, M.M.; Lang, P.J. Affective reactions to acoustic stimuli. Psychophysiology 2000, 37, 204-215. [CrossRef]

20. Anttonen, J.; Surakka, V. Emotions and heart rate while sitting on a chair. In Proceedings of the SIGCHI Conference on Human Factors in Computing Systems; ACM Press: Portland, OR, USA, 2005; pp. 491-499. [CrossRef]

21. Codispoti, M.; De Cesarei, A. Arousal and attention: Picture size and emotional reactions. Psychophysiology 2007, 44, 680-686. [CrossRef]

22. Sokhadze, E.M. Effects of Music on the Recovery of Autonomic and Electrocortical Activity After Stress Induced by Aversive Visual Stimuli. Appl. Psychophysiol. Biofeedback 2007, 32, 31-50. [CrossRef]

23. Task Force of the European Society of Cardiology and Others. Heart Rate Variability: Standards of Measurement, Physiological Interpretation, and Clinical Use. Circulation 1996, 93, 1043-1065. [CrossRef]

24. Gramann, K.; Schandry, R. Psychophysiologie: Körperliche Indikatoren Psychischen Geschehens, 4th ed.; vollst. überarb. aufl, Ed.; Beltz PVU: Weinheim, Germany, 2009.

25. Hare, R.; Wood, K.; Britain, S.; Shadman, J. Autonomic Responses to Affective Visual Stimulation. Psychophysiology 1970, 7, 408-417. [CrossRef] 
26. Libby, W.L.; Lacey, B.C.; Lacey, J.I. Pupillary and Cardiac Activity During Visual Attention. Psychophysiology 1973, 10, $270-294$. [CrossRef]

27. Winton, W.M.; Putnam, L.E.; Krauss, R.M. Facial and autonomic manifestations of the dimensional structure of emotion. J. Exp. Soc. Psychol. 1984, 20, 195-216. [CrossRef]

28. Lang, P.J.; Greenwald, M.K.; Bradley, M.M.; Hamm, A.O. Looking at pictures: Affective, facial, visceral, and behavioral reactions. Psychophysiology 1993, 30, 261-273. [CrossRef]

29. Müller, R.; Büttner, P. A critical discussion of intraclass correlation coefficients. Stat. Med. 1994, 13, 2465-2476. [CrossRef]

30. Pinna, G.; Maestri, R.; Torunski, A.; Danilowicz-Szymanowicz, L.; Szwoch, M.; La Rovere, M.; Raczak, G. Heart rate variability measures: A fresh look at reliability. Clin. Sci. 2007, 113, 131-140. [CrossRef] [PubMed]

31. Portney, L.G.; Watkins, M.P. Foundations of Clinical Research: Applications to Practice, 3rd ed.; Pearson/Prentice Hall: Upper Saddle River, NJ, USA, 2009.

32. Lee, J.; Koh, D.; Ong, C.N. Statistical evaluation of agreement between two methods for measuring a quantitative variable. Comput. Biol. Med. 1989, 19, 61-70. [CrossRef]

33. McGraw, K.O.; Wong, S.P. Forming inferences about some intraclass correlation coefficients. Psychol. Methods 1996, 1, 30-46. [CrossRef]

34. Shrout, P.E.; Fleiss, J.L. Intraclass correlations: Uses in assessing rater reliability. Psychol. Bull. 1979, 86, 420-428. [CrossRef] [PubMed]

35. Altman, D.G.; Bland, J.M. Measurement in Medicine: The Analysis of Method Comparison Studies. Statistician 1983, $32,307$. [CrossRef]

36. Bland, J.M.; Altman, D.G. Comparing Methods of Clinical Measurement-A Citation-Classic Commentary on Statistical-Methods for Assessing Agreement between 2 Methods of Clinical Measurment. Curr. Contents 1992, 40, 8.

37. Ryan, T.P.; Woodall, W.H. The most-cited statistical papers. J. Appl. Stat. 2005, 32, 461-474. [CrossRef]

38. Bland, J.M.; Altman, D.G. Agreement Between Methods of Measurement with Multiple Observations Per Individual. J. Biopharm. Stat. 2007, 17, 571-582. [CrossRef]

39. Shapiro, S.S.; Wilk, M.B. An Analysis of Variance Test for Normality (Complete Samples). Biometrika 1965, 52, 591. [CrossRef]

40. Bland, J.M.; Altman, D.G. Measuring agreement in method comparison studies. Stat. Methods Med. Res. 1999, 8, 135-160. [CrossRef]

41. Weippert, M.; Kumar, M.; Kreuzfeld, S.; Arndt, D.; Rieger, A.; Stoll, R. Comparison of three mobile devices for measuring R-R intervals and heart rate variability: Polar S810i, Suunto t6 and an ambulatory ECG system. Eur. J. Appl. Physiol. 2010, 109, 779-786. [CrossRef]

42. Sandercock, G.R.H.; Shelton, C.; Bromley, P.; Brodie, D.A. Agreement between three commercially available instruments for measuring short-term heart rate variability. Physiol. Meas. 2004, 25, 1115-1124. [CrossRef]

43. Nunan, D.; Donovan, G.; Jakovljevic, D.G.; Hodges, L.D.; Sandercock, G.R.H.; Brodie, D.A. Validity and Reliability of Short-Term Heart-Rate Variability from the Polar S810. Med. Sci. Sport. Exerc. 2009, 41, 243-250. [CrossRef]

44. Da Silva, H.P.; Carreiras, C.; Lourenço, A.; Fred, A.; das Neves, R.C.; Ferreira, R. Off-the-person electrocardiography: Performance assessment and clinical correlation. Health Technol. 2015, 4, 309-318. [CrossRef] 